Revista de Matemática: Teoría y Aplicaciones 2005 12(1 \& 2) : 173-186

CIMPA - UCR - CCSS ISSN: 1409-2433

\title{
MODELO MULTIFRACTAL APLICADO AL RIEGO
}

\author{
José Roberto Mercado E.* Álvaro Alberto Aldama R. ${ }^{\dagger}$ \\ Mauro Íñiguez C..$^{\ddagger}$ Miguel Ángel Mejía G. ${ }^{\S}$
}

Recibido/Received: $17 \mathrm{Feb} 2004$

\begin{abstract}
Resumen
Definimos la distribución multifractal binomial, como expresión de una ley de los pequeños números.

Estudiamos una forma de evaluación o de diseño de un sistema de distribución en un distrito de riego, al determinar su capacidad de conducción con la flexibilidad deseada, conociendo los parámetros de gasto, frecuencia y tiempo de riego. El método es la aproximación de la distribución binomial por la Gaussiana, una relación inversa de probabilidad, dos estimaciones de parámetros Gaussianos, y la aplicación de la distribución multifractal.

Presentamos la reformulación matemática de la relación de Boltzmann en la mecánica estadística, que da origen al modelo multifractal. En particular, ilustramos su aplicación en el problema del riego. Pero también es posible aplicarlo a los modelos multifractales: variograma, exponencial, gama, y Gaussiano.
\end{abstract}

Palabras clave: Riego, flexibilidad, multifractales, binomial, Student, estimación de parámetros.

\footnotetext{
Abstract

We define the binomial multifractal distribution as an expression of a law of small numbers.

We studied a form of evaluation or design of a distribution system in an irrigation district by determining its conduction capacity with the desired flexibility knowing the following parameters: flow, frequency, and irrigation time. The method consists of the approximation of the binomial distribution through the Gaussian, an inverse

*Instituto Mexicano de Tecnología del Agua, IMTA, Paseo Cuauhnáhuac 8532, Progreso, Jiutepec, Mor., C. P. 62550, México; Fax: +\{52\} 77732936 70; E-Mail: rmercado@tlaloc.imta.mx.

${ }^{\dagger}$ Misma dirección que J.R. Mercado. E-Mail: aaldama@tlaloc.imta.mx.

${ }^{\ddagger}$ Misma dirección que J.R. Mercado. E-Mail: mic@tlaloc.imta.mx.

${ }^{\S}$ Misma dirección que J.R. Mercado. E-Mail: mamejia@tlaloc.imta.mx.
} 
174 J.R.MerCAdo - Á.AldAMA - M.ÍÑ̃IgUeZ - M.MeJÍA Rev.Mate.Teor.Aplic. (2005) 12 (1 \& 2)

relation of probability, two estimations of Gaussian parameters, and the application of the multifractal distribution.

We present the mathematical reformulation of Boltzmann relation in the statistical mechanics, which gives origin to the multifractal model. In particular, we illustrate its application in the problems of irrigation, pointing out that it is also possible to apply it to multifractal models: variogram, exponential, gamma, and Gaussian.

Keywords: Irrigation, flexibility, multifractals, binomial, Student, parameter estimation

Mathematics Subject Classification: 28A80, 62E17, 60F05.

\section{Introducción}

El objetivo del presente trabajo es la revisión del modelo multifractal, para introducir la distribución multifractal, y aplicarla a la estimación de parámetros en la determinación del posible número de tomas abiertas, como una forma de valorar y diseñar un sistema de riego.

Los sistemas de distribución, en general, cuentan con muchos puntos de entrega o de recepción; los que a su vez, tienen la posibilidad de estar muy esparcidos en un vasto territorio. Imaginemos por ejemplo, sistemas de distribución de mercancías o productos comerciales, sistemas de distribución de los servicios de agua, luz o teléfono. Sistemas de distribución de presupuestos. Un sistema de votación. Sistemas de recolección o de recaudacción. Las cuencas hidrológicas. Los sistemas de distribución de la energía de las partículas en los átomos y moléculas. El sistema de intercambio entre los pulmones y las células. Estos sistemas ofrecen la posibilidad de describirse a través del modelo de la estructura arbórea fractal.

Veamos entonces, las fuentes teóricas que alimentan el sustento de los modelos del presente trabajo. Por una parte se considera la reformulación matemática de la relación de Boltzmann en la mecánica estadística, que da origen al modelo multifractal, y en particular, al modelo de las pruebas independientes y homogéneas de Bernoulli que dan lugar a la distribución binomial. Y desde otro ángulo, definimos la distribución multifractal, mostramos que esta distribución se aproxima a una distribución student.

El trabajo está organizado como sigue: Realizamos primero, en la sección 2, la reformulación matemática del modelo multifractal partiendo de los conceptos de la mecánica estadística, y en particular trabajando el modelo multifractal binomial. Se describe luego en la sección 3, un sistema de riego como el de la Begoña, Gto. Se clarifica la diferencia de lo que entendemos por el problema directo e inverso en este contexto. Repasamos el teorema del límite central, y la estimación de parámetros Gaussianos, y se elaboran los cuadros donde se determina el número de tomas abiertas con sus respectivos intervalos de confianza. El primero de éstos, se construye con la aplicación de la distribución multifractal inversa. Finalmente, en la sección 4, se presentan las conclusiones. 


\section{Modelo Multifractal}

Un sistema de distribución lo imaginamos como una serie de puntos en el espacio que represente el conjunto de puntos de entrega, el cual presentaría un aspecto esparcido e irregular; los cubrimos con una serie de cubos de un mismo tamaño variable y queremos determinar su dimensión fractal. Además los pintamos con dos colores: uno de color rojo, para los puntos abiertos, y otros de color negro, para los cerrados; y si se encuentra una manera de estimar la probabilidad de que un punto esté iluminado con color rojo, conociendo el número de los puntos iluminados, podemos calcular la probabilidad acumulada; sin embargo, resultaría más interesante tener una manera de estimar cuál es el número más probable de puntos iluminados, dado que la probabilidad acumulada no supere un valor preestablecido.

El estado microscópico de cada toma, se especifica por 0 si está cerrada; y por 1 si está abierta la toma, de tal manera que los estados del sistema se representan por sucesiones del grupo bicíclico $\{0,1\}$, entonces todos los posibles estados del sistema son homeomorfos a un conjunto de Cantor.

Este conjunto, es más conocido cuando se construye por un proceso iterativo que parte de una medida unitaria; se la divide en tres partes iguales y se suprime el tercio intermedio, de tal manera que resultan 2 partes o rasgos de medida $1 / 3$, al final de la primera etapa, y $1(1 / 3)$ de medida desechada. En la segunda, cada una de los dos rasgos se divide nuevamente en tres partes iguales y se desechan los dos tercios intermedios, resultando ahora $2^{2}$ rasgos de medida $1 / 3^{2}$, cada uno, y $2(1 / 3)^{2}$ de la medida desechada; y el proceso se continúa de forma interminable, [1], [4].

En el nivel microscópico tomamos un conjunto de Cantor, como el soporte de la medida micro $\mu$, la cual debe definirse para cada contexto específico. Se conforma luego una malla como una colección de cubos $\left(C_{i}\right)_{i}$ de lado $b$, con $0<1 / b<1$, que intersecten el soporte de la medida $\mu$; y a $b$, se le denomina la resolución.

Por ejemplo la medida de probabilidad $\mu$ puede escogerse como la binomial, la cual se define por la probabilidad de éxitos en un esquema de Bernoulli: $\mu_{k}=p^{k}(1-p)^{n-k}$, siendo $p$ la probabilidad de éxito. Se representan por $b_{k}$ las resoluciones evaluadas en los cubos $C_{k}$ y por $\mu_{k}$ la medida valorada en $C_{k}$, asociada al estado particular $k$, como proporcional a la resolución correspondiente, por

$$
\mu_{k}=\mu\left(C_{k}\right)=\text { cteb }_{k}=p^{k}(1-p)^{n-k},
$$

se tiene así especificada la configuración microscópica, de tal manera que a cada estado microscópico quede asociada una única configuración microscópica.

Se pasa ahora al nivel macroscópico. Correspondientes a los distintos factores de Boltzmann, se definen las configuraciones macroscópicas como proporcionales a cierta potencia de las resoluciones

$$
\mu_{k}^{s}=\operatorname{cteb}_{k}^{s}=\left(\begin{array}{c}
n \\
k
\end{array}\right)\left(p^{k}(1-p)^{n-k}\right)^{s}
$$


176 J.R.MercAdo - Á.AldAMA - M.ÍÑ̃gUeZ - M.MeJÍA Rev.Mate.Teor.Aplic. (2005) 12 (1 \& 2)

Se define la función de partición como la suma total de las configuraciones, o macroconfiguraciones, disponibles del sistema

$$
S_{b}(s)=\sum_{k} \mu_{i}^{s}=\sum_{k}\left(\begin{array}{c}
n \\
k
\end{array}\right)\left(p^{k}(1-p)^{n-k}\right)^{s},
$$

en donde la suma se extiende sobre aquellos cubos que intersectan el soporte de la medida $\mu$ y $S_{b}(0)=a_{b}(\operatorname{sop} \mu)$ representa el número de cubos -de lado $1 / b$ - necesarios para cubrir el soporte de $\mu$.

Se busca una relación de potencia para la función de partición

$$
S_{b}(s) \approx b^{\tau(s)} .
$$

Una vez que se cuenta con la función de partición, se define la distribución de Gibbs, que puede verse como la forma en que se distribuyen las probabilidades de los estados microscópicos dentro de un cuerpo macroscópico; o bien, como la probabilidad de encontrar el estado con micro-configuración medida por $\mu_{k}$, como el número de macro-configuraciones que representan este estado sobre el número total de configuraciones disponibles en el sistema, por

$$
\nu_{k}=\frac{\mu_{k}^{s}}{S_{b}(s)}
$$

pero además, admite también la interpretación de la frecuencia relativa de ocupación de un estado microscópico, [9].

En general el objetivo multifractal es encontrar la función $f$ que varía con el parámetro $\alpha$, el cual recorre todos los valores reales, este parámetro es conocido como exponente Hölder, y resulta estar relacionado con la energía, por lo que es un parámetro energético. La función $f$ por lo general se asume cóncava, diferenciable y estrictamente positiva; y se le conoce como el espectro multifractal, [14], [5].

Por otra parte, cuando se tiene definida la medida $\nu_{k}$, contamos con la opción de pasar a la entropía de Shannon, en donde se toma a la potencia $s$ como parámetro, [6], $f(\alpha)=-\sum_{k} \nu_{k} \ln \nu_{k}$. Sin embargo, en las aplicaciones es frecuente, como es el caso de la binomial, que sea más fácil seguir un camino indirecto, y se deba calcular primero la potencia de la función de partición, para luego hallar el espectro multifractal $f(\alpha)$. La idea es buscar una relación análoga a la que Boltzmann postuló entre entropía y número de configuraciones, [9].

Se consideran los cubos con medida del orden de la potencia $\alpha$, con $\varepsilon$ arbitrario, positivo y pequeño; y los rasgos como el número de cubos en donde la medida sea razonablemente grande, es decir, del orden de $\alpha$, con $1 / b$ pequeño,

$$
\left(\frac{1}{b}\right)^{\alpha+\varepsilon} \leq \mu\left(C_{k}\right)<\left(\frac{1}{b}\right)^{\alpha}, \quad a_{b}(\alpha)=\#\left\{k: \mu\left(C_{k}\right) \geq\left(\frac{1}{b}\right)^{\alpha}\right\} .
$$

Se busca que los rasgos sean del orden de $f(\alpha)$, o lo que es lo mismo, que los rasgos $a_{b}(\alpha)$ obedecen una ley de potencia, cuando la resolución vaya a cero, o con más precisión en 
términos de $\varepsilon$,

$$
a_{b}(\alpha) \approx b^{f(\alpha)}, \quad a_{b}(\alpha+\varepsilon)-a_{b}(\alpha-\varepsilon) \approx b^{f(\alpha)} .
$$

En el formalismo multifractal la potencia - $\tau(s)$-, análoga a la energía libre de Gibbs, resulta ser la transformada de Legendre del espectro multifractal - $f(\alpha)$-, y el máximo se alcanza cuando la curva cóncava $f(\alpha)$ está por encima y lo más alejado posible de la recta $s \alpha$ de pendiente $s$, luego

$$
\tau(s)=\sup _{\alpha \geq 0}\{f(\alpha)-s \alpha\}, \quad \tau(s)=f(\alpha(s))-s \alpha(s) .
$$

Por la condición de máximo, se obtiene que la potencia $s$ es la pendiente del espectro $f$; y como además para el caso presente, $\tau$ es diferenciable, la pendiente de $\tau$ es $-\alpha$,

$$
\frac{d}{d \alpha}(f-s \alpha)=\frac{d}{d \alpha} f-s=0, \quad \frac{d}{d s} \tau=\left(\frac{d}{d \alpha} f \frac{d}{d s} \alpha-s \frac{d}{d s} \alpha\right)-\alpha=0-\alpha .
$$

En particular, el valor nulo del parámetro potencia corresponde al máximo del espectro,

$$
f(\alpha(0))=\tau(0), \quad S_{b}(0)=a_{b}(\operatorname{sop} \mu) \approx b^{\tau(0)} .
$$

Por tanto, $\alpha$ en efecto, admite la comparación con la energía interna, el espectro $f$ con la entropía y la potencia $s$ con el inverso de la temperatura de un sistema termodinámico.

La potencia de la función de partición, en el caso binomial se calcula directamente de la expansión del binomio de Newton y se da en la ecuación (1). Se observa que para el parámetro potencia unidad el exponente de la función de partición es nulo: si $s=1$, $\tau(1)=0$; en tanto que para el valor nulo del parámetro $s$, se tiene la dimensión del soporte de la medida: si $s=0, \tau(0)=f(\alpha(0))=\operatorname{dim}_{B}(\operatorname{sop} \mu)=D_{B}$.

$$
\tau(s)=\log _{b}\left(p^{s}+(1-p)^{s}\right) .
$$

Ahora se halla la pendiente $\alpha$ de la potencia $\tau$, de la función de partición $S_{b}(s)$,

$$
\alpha(s)=-\frac{p^{s} \log _{b} p+(1-p)^{s} \log _{b}(1-p)}{p^{s}+(1-p)^{s}},
$$

por tanto, de $f(\alpha(s))=\tau(s)+s \alpha(s)$, el espectro en forma paramétrica es

$$
f(\alpha(s))=\log _{b}\left(p^{s}+(1-p)^{s}\right)+s\left(-\frac{p^{s} \log _{b} p+(1-p)^{s} \log _{b}(1-p)}{p^{s}+(1-p)^{s}}\right) .
$$

En particular, la multifractal binomial para la probabilidad del proceso de Bernoulli de $p=1 / 7, \operatorname{con} g(r)=\tau(r)+r \alpha(r)$, es:

$$
\tau(r)=\frac{1}{\ln 3} \ln \left(\left(\frac{1}{7}\right)^{r}+\left(\frac{6}{7}\right)^{r}\right), \quad \alpha(r)=-\frac{1}{\ln 3}\left(\frac{\left(\frac{1}{7}\right)^{r} \ln \left(\frac{1}{7}\right)+\left(\frac{6}{7}\right)^{r} \ln \left(\frac{6}{7}\right)}{\left(\frac{1}{7}\right)^{r}+\left(\frac{6}{7}\right)^{r}}\right) .
$$


178 J.R.MerCAdo - Á.AldAMA - M.Íñ̃IGUEZ - M.MeJíA Rev.Mate.Teor.Aplic. (2005) 12 (1 \& 2)

A partir de este espectro multifractal se define la distribución multifractal $M(x)$ :

$$
M\left(x ; \frac{1}{7}\right)=\frac{1}{1.6713} \int_{-\infty}^{x} f(r) d r
$$

porque se verifican las condiciones: $f(r) \geq 0$, para casi todo $r$; que $M(\infty) \rightarrow 1$; y además se observa que $f(r)$ es medible según Lebesgue, entonces $f(r)$ define una densidad de cierta magnitud aleatoria.

Por otra parte, la probabilidad de que la magnitud aleatoria no supere cierto valor, en el caso de la conocida distribución student o T, se da por la expresión

$$
\operatorname{Pr}(\xi \leq x)=\operatorname{TDist}(x ; v)=\frac{\Gamma\left(\frac{v+1}{2}\right)}{\Gamma\left(\frac{v}{2}\right) \sqrt{\pi v}} \int_{-\infty}^{x}\left(1+\frac{1}{v} s^{2}\right)^{-\frac{v+1}{2}} d s,
$$

donde $\Gamma$ es la función delta de Euler; y al parámetro de forma $\nu$, se le conoce como los grados de libertad. Para el caso presente este número es igual a $5-1=4$, luego

$$
\operatorname{Pr}(\xi \leq x)=\operatorname{TDist}(x ; 4)=\frac{3}{8} \int_{-\infty}^{x}\left(1+\frac{1}{4} s^{2}\right)^{-\frac{5}{2}} d s .
$$

Las gráficas de la dos distribuciones se presenta en la (figura 2), con línea continua para la multifractal y con pequeños círculos para la Student. Las densidades también se muestran en la figura (1), la multifractal con línea continua, la Student con pequeños círculos y la Gaussiana con cruces. En esta gráfica se observa que las tres densidades tienen la forma de campana, son simétricas, y sus medias son cero. Comparadas con la Gaussiana, la multifractal y Student tienen menor altura, mayor varianza, y las colas son más grandes. En particular, tanto la distribución multifractal como la Student, son características de ley de los pequeños números, y son útiles cuando los tamaños de las muestras son pequeños. Aclaramos que el valor $\nu=4$, para los grados de libertad se ha escogido, para que el tamaño de la muestra sea pequeña, en tanto se ha encontrado que para el valor $p=\frac{1}{7}$, $b=3$, se obtiene la proximidad citada entre las dos distribuciones.

$\mathrm{Al}$ comparar el espectro multifractal como función del parámetro potencia con la distribución Student, la representación gráfica de las dos nos muestra la cercanía de una con la otra. Esta cercanía también se refuerza cuando se observan y comparan las dos expansiones en serie. Se ilustra la comparación entre la multifractal binomial con probabilidad del proceso de Bernoulli de $p=1 / 7, b=3$, con la Student de 4 grados de libertad, en la gráfica (2) para las distribuciones y, en (1) para las densidades.

\section{$3 \quad$ El problema del riego}

Un sistema de distribución de agua para riego se describe como un canal inicial, con mayor capacidad de conducción del agua, luego una secuencia de canales laterales cada vez de menor capacidad de conducción, hasta llegar a las tomas, en las cuales se entrega 


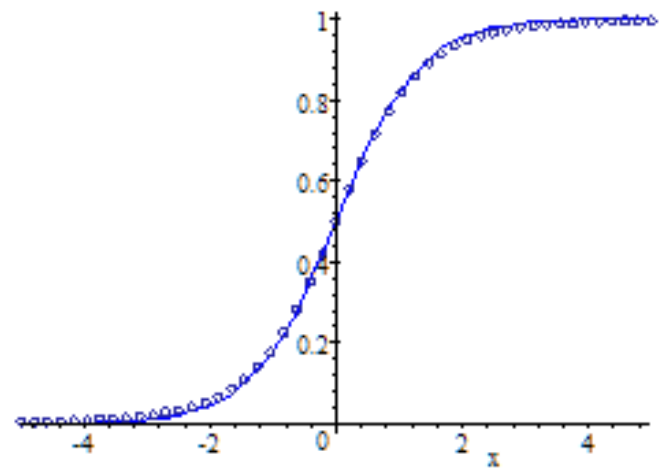

Figura 1: Distribución Multifractal y Student.

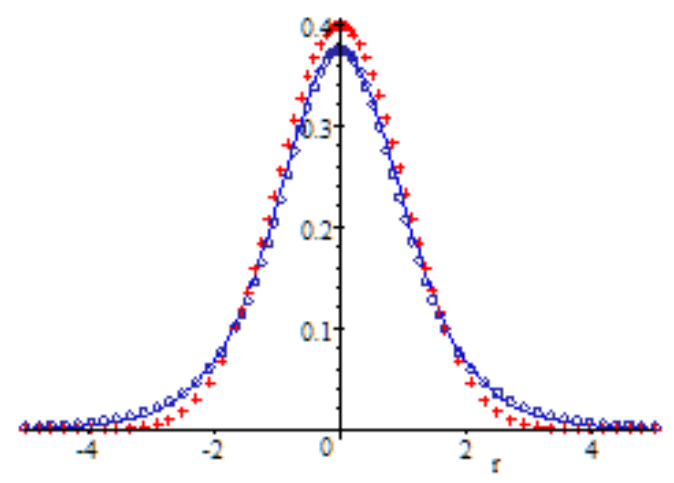

Figura 2: Densidad Multifractal, 1/7; Student, 4; Gaussiana.

el agua para el riego en los surcos de las parcelas. Un perfecto modelo de este sistema de distribución, es un árbol; con un tronco inicial de mayor diámetro, que progresivamente se abre en ramas de menor diámetro hasta llegar a las hojas, como se muestra en la figura (3) ${ }^{1}$.

En el caso del distrito de riego de la Begoña, Gto., a partir de una presa de mayor capacidad, llamada la presa derivadora, se van formando una secuencia de canales laterales con la posibilidad de representarse en forma de un árbol, hasta llegar al nivel de las tomas, en donde se entrega el agua para los surcos en las parcelas. Cada nodo de ese árbol se interpreta como un nodo inicial similar al de la derivadora, de tal modo que en cada nodo se inicia un subárbol, y el número de tomas $n$ se ve como una sucesión de estos nodos iniciales: $n(i)=n_{i}$. Y en particular para el distrito de la Begoña, se consideran 10 subárboles, así que el subíndice $i$ varía desde 1 hasta 10.

\subsection{Problema directo e inverso}

En el sistema de distribución de agua para riego, los puntos de entrega los representamos con dos colores: uno de color rojo, para los puntos abiertos, y otros de color negro, para

\footnotetext{
${ }^{1}$ Según diseño de Martha Morales Martínez.
} 


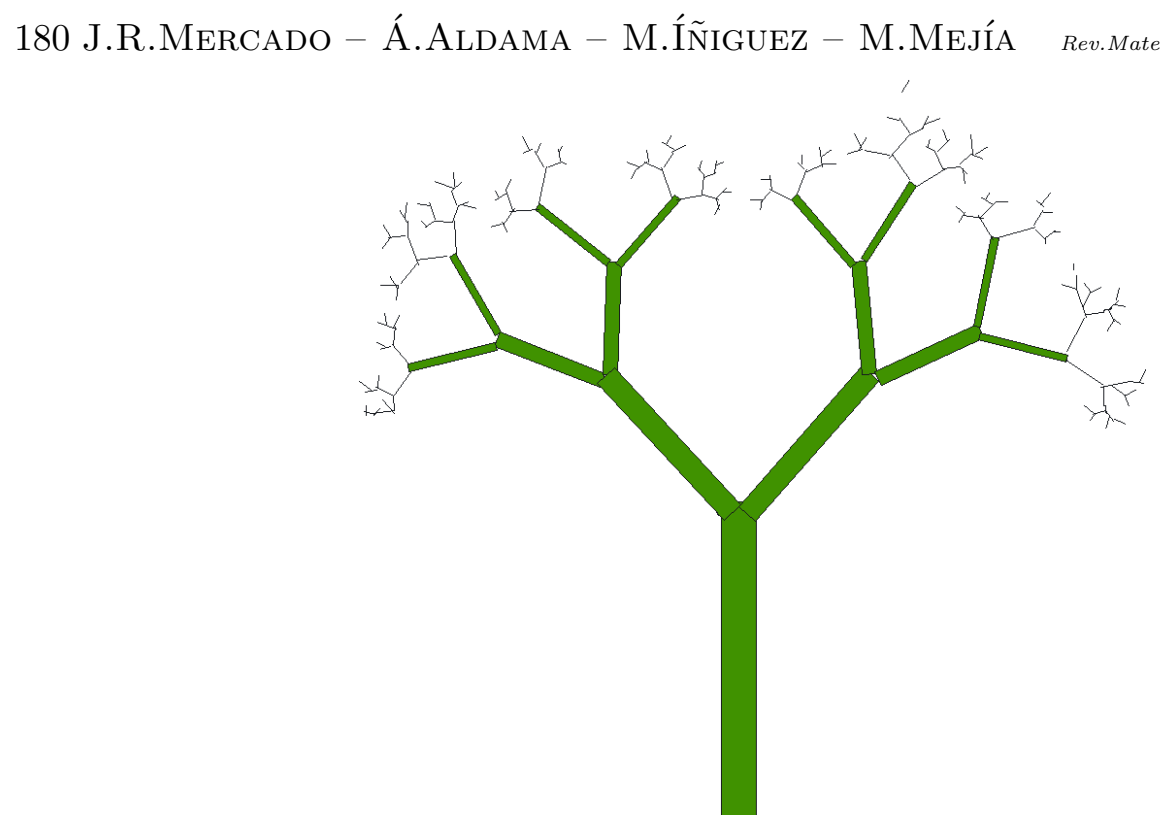

Figura 3: Estructura arbórea.

los cerrados. El constatar si una determinada toma está abierta, se concibe como un experimento aleatorio, así que los posibles resultados de los experimentos son 1 o 0; si está abierta, se ilumina de rojo y la magnitud aleatoria asume el valor 1; y en el caso contrario, se pinta con negro y el valor es 0 .

Como hay un total de $n$ tomas, se obtiene un experimento compuesto de $n$ pruebas, las cuales son independientes y del mismo tipo para todas las tomas. Se componen las $n$ magnitudes aleatorias, una por cada toma, para formar la magnitud aleatoria suma $\xi$, la cual se describe afirmando que del total de las $n$ tomas, $k$ se encuentran abiertas y $n-k$ están cerradas.

La medida de probabilidad del estado abierto se define como $p$, y al estado cerrado como $q=1-p$. Al resultado del experimento aleatorio compuesto se le asocia un valor de la medida de probabilidad dada por $B_{\xi}$, y la probabilidad de que haya $m$ tomas abiertas es entonces la suma de probabilidades

$$
B_{\xi}(n, k ; p)=\left(\begin{array}{c}
n \\
k
\end{array}\right) p^{n-k} q^{k}, \quad \sum_{k=0}^{m} B_{\xi}(n, k ; p)=P_{p}(n, m),
$$

que se conoce como un modelo probabilístico binomial de pruebas de Jacob Bernoulli.

De acuerdo con la ley de los grandes números una sucesión de magnitudes aleatorias independientes e igualmente distribuidas, en el límite tendrá, a la uniforme, como su distribución, independientemente de cuál haya sido su distribución a lo largo de todo su proceso evolutivo, [3]. Entonces, la desviación cuadrática media se da por la distancia entre el valor estimado y el valor teórico [15],

$$
\left\|\frac{n(A)}{n}-p\right\|=\frac{1}{n} \sqrt{n p q}=\sqrt{\frac{p q}{n}}
$$


En los sistemas de riego se aplican los métodos: de distribución continua, de demanda libre, de demanda controlada y de rotación, turno o tandeo. Si $Q_{r o t}$ denota el gasto de rotación, $r$ la fracción de tiempo de ocupación del sistema, $n$ el número de tomas, y $Q_{T}$ el gasto en cada toma. La manera de estimar la probabilidad de que un punto esté iluminado con color rojo o que una toma esté abierta, $p$, consiste en avalúar

$$
p=\frac{Q_{\text {rot }}}{r n Q_{T}},
$$

Por tanto, el problema directo se enuncia diciendo que: se tiene la densidad de probabilidades, y luego se integra para obtener la probabilidad acumulada. Si todos los puntos de entrega están abiertos, la probabilidad acumulada tiene que ser 1, por construcción.

En cambio para el problema inverso se tiene: dada una probabilidad acumulada, cuál es el número más probable de puntos de entrega que están coloreados de rojo? Éste es el problema que se aborda, dada la probabilidad $P_{p}$, se quiere conocer cuál es el número $m$ más probable de tomas que estén abiertas, [11].

Invertir la probabilidad dada por la binomial no es posible porque ésta es una distribución discreta y tal operación carece de sentido, sin embargo, cuando el número de pruebas $n$ es suficientemente grande, es posible aproximar la distribución binomial por la distribución normal de Gauss, a través del expediente de embeber o diluir la binomial en la normal, tal como fue demostrado por Abraham de Moivre, en 1733, para el caso particular $p=1 / 2$.

Los factoriales presentes en la distribución binomial surgen de las diversas formas en que los objetos de cierta muestra se pueden agrupar en submuestras de objetos indistinguibles, y para un gran tamaño de muestra éstos crecen en forma descomunal. El teorema de deMoivre-Laplace, en su forma intuitiva, se basa en aproximar el logaritmo y los factoriales; para el primero, se usa la expansión de Taylor; y para los segundos, se recurre a la aproximación del escocés, James Stirling, de 1730, para los factoriales de los grandes números, que permite convertir estos factoriales en potencias.

A grandes rasgos, se trata de tomar algún intervalo como: $\left[\frac{-n p}{\sqrt{n p q}}, \frac{n q}{\sqrt{n p q}}\right]$, dividirlo en $n$ intervalos iguales de longitud $\Delta x=\frac{1}{\sqrt{n p q}}$, con extremos $x_{n, k}$; definir la magnitud aleatoria normalizada $\xi_{n}$ que toma uno de estos valores $x_{n, k}$ de la forma

$$
x_{n, k}=\frac{k-n p}{\sqrt{n p q}}, k=0,1, \ldots n
$$

se calcula la probabilidad acumulada de la binomial; se usan las aproximaciones, y se obtiene la distribución Gaussiana $G$ :

$$
P_{p}(n, m)=\sum_{k=0}^{m \leq n} B_{\xi}(n, k ; p)=\sum_{k=0}^{m}\left(\begin{array}{c}
n \\
k
\end{array}\right) p^{n-k} q^{k} \simeq G\left(x_{n, m}\right) ;
$$

como la función de distribución es inyectiva tiene inversa por la izquierda, y finalmente se obtiene

$$
G^{-1}\left(P_{p}(n, m)\right)=x_{n, m}=\frac{m-n p}{\sqrt{n p q}}, \quad m=n p+G^{-1}\left(P_{p}(n, m)\right) \sqrt{n p q} .
$$


182 J.R.MerCADO - Á.AldAMA - M.ÍÑ̃IGUEZ - M.MeJíA Rev.Mate.Teor.Aplic. (2005) 12 (1 \& 2)

Por otra parte, en este trabajo encontramos la cercanía de la función del espectro, en tanto función del parámetro potencia, con la distribución student, y es conocida la cercanía de la distribución student con la Gaussiana:

$$
M\left(x_{n, m}\right) \simeq S\left(x_{n, m}\right), \quad S\left(x_{n, m}\right) \simeq G\left(x_{n, m}\right)
$$

las tres densidades se ilustran en la figura 2 .

\subsection{Estimación de parámetros}

La extensión y diversidad en los sistemas de distribución, obliga a plantearse métodos de clasificación para evaluar un sistema. En el caso de la distribución del agua con fines de riego, la idea de Clement consiste en determinar, según la forma de la estimación de los parámetros y el número de tomas, sólo dos clases : a) la estimación de la media con desviación desconocida para un número mayor que 100 tomas; y b) la estimación de la media con desviación conocida, para un número menor que 100 tomas. Por tanto, la relación inversa se despliega en dos ecuaciones, en razón de la naturaleza de las estimaciones, [2].

\subsubsection{La clase central}

Para la clase con un número mayor que 100 tomas, se tienen, para el primer árbol desde la presa derivadora, 384 observaciones independientes posibles de la magnitud aleatoria Gaussiana, en donde el parámetro que se estima es la media Gaussiana con varianza desconocida, luego es de esperar una cola mayor. Se sabe que en estos casos se debe recurrir a la ley de los pequeños números, o tamaños de muestra de números pequeños, de William Sealy Gosset, quien usó el seudónimo de student, (1908), [12]; pero debido a la cercanía mostrada en este trabajo con la multifractal binomial, ésta igualmente expresa una de las leyes de los pequeños números, y en consecuencia también es susceptible de ser usada con el mismo propósito.

Dentro de una muestra de $m$ tomas, un usuario en particular demanda el servicio, la razón entre la probabilidad que desde cualquiera de las tomas lo encuentre disponible, y la probabilidad que así sea desde su propia toma, es el índice de congestión, $P_{a}$. Se fija un índice de congestión relativamente bajo, del orden del 1\%, con lo cual se pretende valorar la disponibilidad del sistema cuando es demandado.

Así, se obtiene una de las fórmulas del modelo propuesto. Se calcula $P_{p}=\sqrt{n p q} P_{a}$, se aplica la distribución multifractal inversa, se evalúa por los valores tabulados de la student inversa, $M^{-1}\left(P_{p}\right) \simeq \operatorname{TInv}\left(P_{p}\right)$, y se obtiene el número de tomas abiertas por

$$
\sqrt{n p q} P_{a}=P_{p}=M\left(x_{n, m}\right), \quad m=n p+M^{-1}\left(P_{p}\right) \sqrt{n p q} .
$$

Para estimar la media Gaussiana se toma un tamaño de muestra de 5 tomas, cercano al 10\%. La hipótesis es $H_{0}$ : la media Gaussiana asume el valor específico de la media muestral: $\mu=\bar{x}$. La media se estima como promedio aritmético de las 5 observaciones, o media muestral, con lo que resulta una estimación insesgada en el sentido, de que la esperanza matemática de las estimaciones coincide con el valor del parámetro que se estima: la media Gaussiana. Como la esperanza de las desviaciones cuadráticas de las 
estimaciones con respecto al parámetro la media Gaussiana, es la $n$-ésima parte de la varianza Gaussiana, los momentos muestrales y teóricos coinciden y la estimación se dice conciliable, [10].

Desde el punto de vista estadístico, una de las distintas formas cuantiles en que se agrupan los datos, es la forma percentil, expresada como porcentaje, o bien como índice. El espacio muestral en este caso es la recta real: $\mathbb{R}$. Se divide el espacio muestral en tres partes estableciendo dos marcas cuantilas: $\pm c(\alpha)$. Por fuera de esas cuantilas quedan las dos colas de la campana multifractal. Se fija el nivel de significación en 0.05, lo que significa que el $5 \%$ del área de la campana, está en las llamadas colas, luego el $2.5 \%$ está en cada una de las colas; por tanto el $95 \%$ está por dentro del intervalo; o en términos de índice, el nivel de confianza es: $\alpha=0.95=1-0.05$, [16].

Desde $-\infty$ hasta la cuantila $c(\alpha)$ extremo del intervalo, se tiene el $95 \%+2.5 \%=97.5 \%$, entonces se calcula la inversa de la distribución multifractal: $M^{-1}(0.975,3) \simeq \operatorname{TInv}(0.975,3)=3.1824=c(0.95), \quad \mathrm{y}$ se tiene la cuantila: $c(\alpha)=c(0.95)=3.1824$. Por tanto, para cada uno de los árboles que se inician en los nodos desde donde salen los canales laterales, y que cuentan con más de 100 tomas, tiene los intervalos de confianza

$$
(n p-3.1824 \sqrt{n p q}, n p+3.1824 \sqrt{n p q}) .
$$

La ecuación (8) determina el gasto en términos del número de tomas $n$, tal que la probabilidad de que a lo más $m$ tomas operen simultáneamente, no supere el nivel de confianza de $95 \%$.

Para la ecuación (8) los resultados se muestran en la tabla 1, llamada clase central, en donde la columna 6, para la probabilidad inversa, se construye con la distribución multifractal inversa. La forma específica de calcular sus valores, se ilustra con más detalle en la subsección de la clase perisférica.

\begin{tabular}{c|ccccccc}
\hline \hline Posición & Área & Gasto & Tomas & $\begin{array}{c}\text { Probabilidad } \\
p\end{array}$ & $\begin{array}{c}\text { Probabilidad } \\
\text { inversa }\end{array}$ & $\begin{array}{c}\text { Tomas } \\
\text { abiertas }\end{array}$ & Binomial \\
\hline $0+000$ & 8132 & 9050.9 & 384 & 0.19642 & 1.81852630 & 90 & 0.96257 \\
$1+612$ & 7000 & 7791.0 & 330 & 0.19674 & 1.86273229 & 78 & 0.96770 \\
$5+485$ & 6000 & 6678.0 & 283 & 0.19664 & 1.90887096 & 68 & 0.97033 \\
$5+460$ & 5000 & 5565.0 & 236 & 0.19650 & 1.96457296 & 58 & 0.97403 \\
$5+460$ & 3500 & 3895.5 & 165 & 0.19674 & 2.07754585 & 43 & 0.98213 \\
$6+758$ & 2500 & 2782.5 & 118 & 0.19650 & 2.18885890 & 32 & 0.98155 \\
\hline \hline
\end{tabular}

Tabla 1: Clase central.

\subsubsection{La clase perisférica}

La probabilidad acumulada para que la magnitud aleatoria no supere cierto valor bajo la curva de la campana de Gauss, se da por la distribución normal, la cual se define, para cualesquiera valores de los parámetros de la media $\mu$ y de la desviación estándar $\sigma$, por la 
184 J.R.MercAdo - Á.AldAMA - M.ÍÑ̃gueZ - M.MejÍA Rev.Mate.Teor.Aplic. (2005) 12 (1 \& 2)

integral

$$
\operatorname{Pr}(\xi \leq x)=G(x ; \mu, \sigma)=\frac{1}{\sigma \sqrt{2 \pi}} \int_{-\infty}^{x} e^{-\frac{(t-\mu)^{2}}{2 \sigma^{2}}} d t .
$$

Las áreas debajo de la curva Gaussiana representan también la probabilidad acumulada. Nuevamente el problema de la estimación de parámetros tiene que ver con los dos parámetros de la distribución normal, la media y la desviación estándar.

Para esta clase, se debe estimar la media Gaussiana con varianza conocida. La hipótesis también es $H_{0}$ : la media Gaussiana asume el valor específico de la media muestral: $\mu=\bar{x}$. Se asume una muestra del tamaño del orden de 30 tomas, se calcula la media muestral o empírica, como promedio aritmético, y ésta resulta entonces insesgada y conciliable.

El espacio muestral también en este caso es la recta real: $\mathbb{R}$, y la densidad de la distribución está representada por la campana de Gauss. Se divide el espacio muestral en tres partes con las dos marcas cuantilas: $\pm c(\alpha)$. Por fuera de esas cuantilas quedan las dos colas de la campana Gaussiana. Se fija el nivel de significación en 0.05, luego el $2.5 \%$ está en cada cola, y el $95 \%$ está por dentro del intervalo; y el nivel de confianza es: $\alpha=0.95=1-0.05$.

Por tanto, desde $-\infty$ hasta la cuantila $c(\alpha)$ extremo del intervalo, se tiene el $95 \%+2.5 \%=97.5 \%$, entonces se calcula la inversa de la distribución Gaussiana estándar: NormalInv $(0.975)=1.96$, y se tiene la cuantila: $c(\alpha)=c(0.95)=1.96$. En consecuencia, los intervalos de confianza son:

$$
(n p-1.96 \sqrt{n p q}, n p+1.96 \sqrt{n p q}) .
$$

La ecuación (6), determina el gasto en términos del número de tomas $n$, tal que la probabilidad de que a lo más $m$ tomas operen simultáneamente, sea más grande que la probabilidad deseada para el nivel de satisfacción de 97.5\%. Al fijar el valor de la cuantila en 1.96 se tiene que la probabilidad acumulada es del $97.5 \%$, con este valor, la posibilidad de que un usuario, al recurrir al sistema, encuentre la disponibilidad del agua para el riego deseada, es casi cierta.

Los cálculos realizados para cada uno de ellos a través de la relación inversa de probabilidad dada por la ecuación (6), se presentan en la tabla llamada 2 clase perisférica, y de acuerdo con el autor [8].

En la primera columna se ilustra la posición de los sucesivos nodos, en donde se inician cada uno de los subárboles, en kilómetros desde la derivadora. En la segunda, se dan las áreas susceptibles de regar. En la tercera, se tiene el gasto o caudal de rotación, en lps, litros por segundo, destinado al riego de cada una de las áreas de la respectiva fila, en donde $1.113 \mathrm{lps} / \mathrm{ha}$ se conoce como gasto unitario por hectárea. En la cuarta, se da el número total de tomas para cada subárbol. En la quinta, se da la probabilidad estimada $p$ en donde

\begin{tabular}{c|c}
\hline \hline$i$ & $Q_{\text {rot }}$ \\
\hline 7 & $1.113 \times 2000=2226.0$ \\
8 & $1.113 \times 1500=1669.5$ \\
9 & $1.113 \times 1000=1113.0$ \\
10 & $1.113 \times 500=556.5$ \\
\hline \hline
\end{tabular}

\begin{tabular}{c|cc}
\hline \hline$i$ & $p=\frac{Q_{\text {rot }}}{r n Q_{T}}$ & \\
\hline 7 & $\frac{2226.0}{1 \times 94 \times 120}$ & $=.19734$ \\
8 & $\frac{1669.5}{1 \times 71 \times 120}$ & $=.19595$ \\
9 & $\frac{1113.0}{1 \times 47 \times 120}$ & $=.19734$ \\
10 & $\frac{556.5}{1 \times 24 \times 120}$ & $=.19323$. \\
\hline \hline
\end{tabular}


En la sexta, se presenta la probabilidad total, de acuerdo con el nivel de significación, por ejemplo, para $i=7$, se calcula la media:

$$
n p=94 \times \frac{2226}{1 \times 94 \times 120}=\frac{2226}{1 \times 120}=18.55,
$$

la desviación:

$$
\sqrt{n p q}=\sqrt{\frac{2226}{1 \times 120}\left(1-\frac{2226}{1 \times 94 \times 120}\right)}=3.8587 \approx 3.859
$$

y en forma análoga para las restantes ${ }^{2}$. En la séptima, se ilustra el número de tomas abiertas probables de acuerdo con la probabilidad inversa. El valor de la cuantila para el nivel de significación de 0.05 es: NormalInv $(.975)=1.96$, luego el número de tomas abiertas de acuerdo con la ecuación (6) es

$$
94 \times .19734+1.96 \sqrt{94 \times .19734 \times(1-.19734)}=26.113 \approx 26 .
$$

En la octava y última, se calculan las probabilidades acumuladas de acuerdo con la distribución binomial, por ejemplo para $i=7$ :

$$
\text { BinomialDist }(26 ; 94,0.19734)=.97678
$$

que deben compararse con la probabilidad acumulada según la Gaussiana, al fijar el nivel de significación en 0.05, o lo que es equivalente, el nivel de confianza en 0.95.

\begin{tabular}{c|ccccccc}
\hline \hline Posición & Área & Gasto & Tomas & $\begin{array}{c}\text { Probabilidad } \\
p\end{array}$ & $\begin{array}{c}\text { Probabilidad } \\
\text { inversa }\end{array}$ & $\begin{array}{c}\text { Tomas } \\
\text { abiertas }\end{array}$ & Binomial \\
\hline $9+587$ & 2000 & 2226.0 & 94 & 0.1974 & 1.96 & 26 & 0.97678 \\
$13+198$ & 1500 & 1669.5 & 71 & 0.19595 & 1.96 & 20 & 0.97130 \\
$14+564$ & 1000 & 1113.0 & 47 & 0.19734 & 1.96 & 15 & 0.96710 \\
$16+420$ & 500 & 556.5 & 24 & 0.19323 & 1.96 & 8 & 0.97050 \\
\hline \hline
\end{tabular}

Tabla 2: Clase perisférica.

\section{Conclusiones}

- La construcción de las tablas contienen el procedimiento para evaluar o determinar la capacidad de conducción de un sistema de riego, con la flexibilidad deseada en gasto, frecuencia y duración; ya sea con el propósito de rehabilitar o de diseñar una red, [8].

- La valoración de un sistema de distribución de riego, para la clase central, puede realizarse a través de la distribución multifractal binomial.

\footnotetext{
${ }^{2}$ Para elaborar la tabla se recurre al programa de Excel, de Microsoft Office.
} 
186 J.R.MerCADO - Á.AldAMA - M.ÍÑ̃IgUeZ - M.MeJíA Rev.Mate.Teor.Aplic. (2005) 12 (1 \& 2)

- El modelo multifractal binomial produce una colección de distribuciones de probabilidad, cuyas densidades tienen forma de campana y poseen dos colas más largas que las Gaussianas.

- La distribución multifractal binomial expresa una ley de los pequeños números.

- Para la probabilidad de éxito de 1/7 en el esquema de Bernoulli, la distribución multifractal binomial es cercana a la distribución student de 4 grados de libertad.

\section{Referencias}

[1] Barnsley, M.F. (1993 Fractals Everywhere. Academic Press Professional, Boston.

[2] Clemmens, A.J. (1986) "Canal capacities for demand under surface irrigation", Journal of Irrigation and Drainage Engineering 112(4): 331-347.

[3] Doob, J.L. (1960) Stochastic Processes. John Wiley \& Sons, New York.

[4] Falcon, L.E. (1996) Tomografía Fractal. Tesis Universidad Nacional Autónoma de México, Facultad de Ciencias, México D.F.

[5] Falconer, K. (1990) Fractal Geometry. John Wiley, New York.

[6] Guiasu, S.; Shenitzer, A. (1985) The Mathematical Intelligencer, Vol. 7 No 1. SpringerVerlag.

[7] Guy, R.K. (1998) "The strong law of small numbers", Amer. Math. Monthly 95: 8.

[8] Íñiguez, M. (1994) Análisis de la Flexibilidad en la Distribución del Agua en los Distritos de Riego. Tesis, Colegio de Postgraduados, México D.F.

[9] Kauzmann, W. (1967) Termodinámica y Estadística, Propiedades Térmicas de la Materia, Vol 2. Ed. Reverté, Barcelona.

[10] Koroliuk, V.S. (1981) Manual de la Teoría de Probabilidades y Estadística Matemática. Ed. Mir, Moscú.

[11] Mercado, J.R.; Aldama, Á.A.; Brambila, F. (2003) "Sobre el problema inverso de difusión", Revista de Matemática: Teoría y Aplicaciones 10(1 \& 2): 92-105.

[12] O'Connor, J.J.; Robertson, E.F.

http://www-history.mcs.st-andrews.ac.uk/history/

[13] Pachepsky, Y. ; Timlin, D. (1998) "Water transport in soils as in fractal media", Journal of Hydrology 204: 98-107.

[14] Riedi, R.H.; Scheuring, I. (1997) "Conditional and relative multifractal spectra", Fractals 5(1): 153-168.

[15] Rozanov, Y. (1973) Procesos Aleatorios. Ed. Mir, Moscú.

[16] Spiegel, M.R. (1970) Teoría y Problemas de Estadística. McGraw-Hill, Panamá. 